\title{
Tim Boland
}

U.S. DEPARTMENT OF COMMERCE Technology Administration

National Institute of Standards

and Technology

Gaithersburg, MD 20899

QC 



\section{ISDN LAN Bridging}

\section{Tim Boland}

U.S. DEPARTMENT OF COMMERCE Technology Administration National Institute of Standards and Technology

Gaithersburg, MD 20899

November 1994

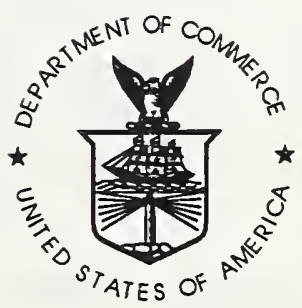

U.S. DEPARTMENT OF COMMERCE Ronald H. Brown, Secretary

TECHNOLOGY ADMINISTRATION Mary L. Good, Under Secretary for Technology

NATIONAL INSTITUTE OF STANDARDS

AND TECHNOLOGY

Arati Prabhakar, Director 


\section{DATE DUE}

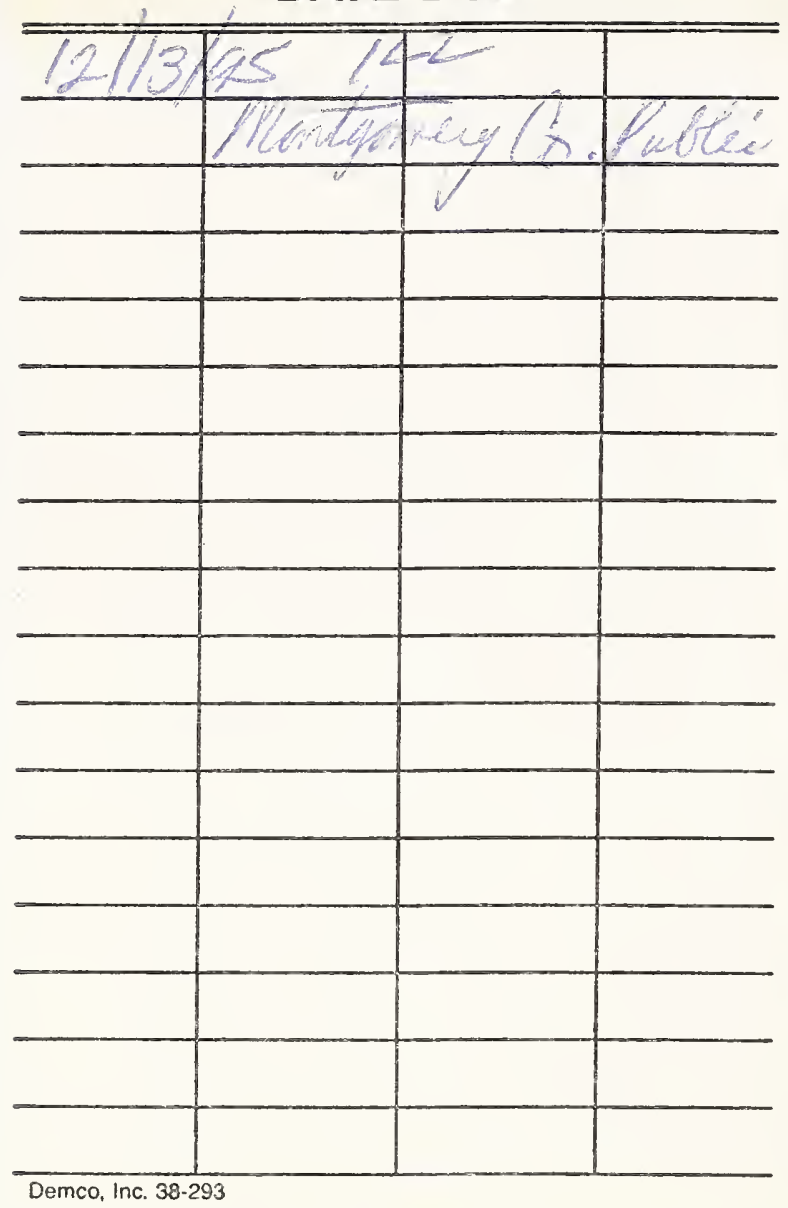




\section{ISDN IAAN BRIDGING}

\subsection{Introduction}

This paper will provide guidance which will enable users to properly assimilate Integrated Services Digital Network (ISDN) local area network (LAN) bridging products into the workplace. This technology is expected to yield economic, functional and performance benefits to users.

Section 1 (this section) provides some introductory information. section 2 describes the environment to which this paper applies. section 3 provides history and status information. Section 4 describes service features of some typical product offerings. section 5 explains the decisions that users have to make and the factors that should influence their decisions. Section 6 deals with current ISDN LAN bridge interoperability activities. Section 7 gives a high-level summary and future direction.

\subsection{ISDN LAN Bridging Environment}

\subsection{User Environment}

ISDN LAN bridge usage should be considered by users who have a need to access a LAN or specific device across a distance of greater than a few kilometers, or by users who are on a LAN and need to access a specific device or another network remotely, and,

for both situations, have or are considering ISDN use to accomplish this access. Thus the interconnection across the ISDN may be LANLAN, point-to-LAN, or point-to-point. The local user environment may consist of one or more LANs or may be single terminals, phones, or fax machines. Users may also have devices called PBXs (private branch exchanges) in their environment; PBXs can handle multiple voice-grade conversations at the same time.

\subsection{Networking Environments}

A I.AN supports multiple users across distances of at most a few kilometers; ownership is usually by a single organization. Data and applications can be shared across a LAN. Adjacent LANs can be connected to create the appearance of a single IAN to a user. BY sharing resources, LANs provide cost savings and performance enhancements to users. The two most common types of LANs used with ISDN LAN bridges are Ethernet (similar to Institute of Electrical and Electronics Engineers (IEEE) standard 802.3) and token ring (IEEE 802.5 ).

An Integrated Services Digital Network (ISDN) can simultaneously transport voice, video, data, or fax in an all-digital environment across long distances at relatively high speeds. This multimedia capability distinguishes ISDNs from other technologies. ISDNS 
provide flexibility, high carrying capacity, and accuracy (low error rates) to users in a wide area networking environment. An ISDN eliminates the need for duplicate lines. Time division multiplexing (use of recurring "time slices") provides sharing of the digital bit pipe by the information types

mentioned above.

All types of devices may be connected to an ISDN (including an ISDN LAN bridge); however, the interfaces or intersections with the ISDN must be uniform, so that the ISDN can provide a high quality of service to disparate users. Different ISDNs can be connected, but this may not be practical, because different ISDN switches are not always compatible.

Basically, ISDN represents a revision or overhauling of the public telephone system. Previously, the telephone system used voicegrade lines: now many of these lines are being digitized. This digitization is resulting in higher speeds and more accuracy and flexibility. Many terms are similar for both ISDN and phone use. For example, an ISDN LAN bridge places a call, just like a telephone user does, and many of the services are analogous to those provided by the phone company. ISDN services may be available by subscription or at the time a call is made.

The carrier may place a network terminating device (NT1) on the customer's premises, and connect it to the carrier's ISDN. The functions of an NT1 device include line transmission termination, line maintenance and performance monitoring, and power transfer. The NTI device (part of the carrier's equipment) provides an interface (network boundary) to the customer's equipment. For larger customer sites, customer equipment may include another type of network terminating device (NT2). An NT2 device is an intelligent device which may provide protocol handling, multiplexing, switching, and concentration functions. An NT2 device may be connected to an NT1 device, and provides the real interface for telephones, terminals, and other user equipment; it aggregates this equipment to provide a consistent interface to the carrier's ISDN.

This paper deals with ISDN LAN bridging with narrowband ISDN - ISDN using copper wire. This limits the speeds which can be achieved, but exploits technology widely available today. In broadband ISDN, fiber is used instead of copper wire. Broadband ISDN supports greater speeds and more functional capability for the user, but it is a technology of the future, and so is not addressed here.

An ISDN recognizes several types of interfaces. Two common ones are the Basic Rate Interface (BRI) and the Primary Rate Interface (PRI). The BRI and PRI support different combinations of two channel types - the two channel types are the B channel (64 Kbps channel for user data) and the $D$ channel (16 or 64 Kbps digital channel for out-of-band signalling). out-of-band signalling 
carries control information (non-user data) for the dialogue. This is fundamentally different from a LAN, where the control information and da ase the same cable.

The BRI uses two B channels plus a D channel, and the PRI in the U.S. uses 23 B channels plus a D channel. The BRI replaces the Plain old Telephone service (POTS) for home or small business use, or for use by indivi lal employees in a large company. The PRI is for larger simulti pous volumes of traffic. The PRI can simultaneously supp $t$ up to 23 users over a single line. In an applications sense, many more end users can be supported, and can benefit from ISDN LAN bridge technology. Other protocols can use the ISDN (among other choices) as a supporting physical medium; for example, the Point-to-Point Protocol (PPP), which negotiates and establishes the characteristics of a line between two fixed end points, can use ISDN in this way, in which case the network is a "PPP network". ISDN LAN bridging applies to this scenario.

ISDN switches are devices that take incoming data in the digital bit pipe and send it on to a further destination. ISDN switches are part of the service provider's equipment, and the kind of switch used is important for ISDN LAN bridging, since not all switches interwork with each other. Some ISDN LAN bridges are connected directly to an ISDN switch (which means that the bridge may have NT2 logic implemented), and some are connected to an NT1 device.

\subsection{ISDN LAN Bridge Definition}

Simple LAN-LAN bridges (such as Ethernet-to-Ethernet bridges) have been in existence for some time. As the name implies, they join two physically adjacent LANs of the same type via a common link to create the appearance of a single LAN to the user. In this way resource sharing is amplified. A bridge of this type forwards frames if appropriate and may amplify the signal between LANs. A frame is a group of data bits with control information included. Since IAN networks have a distance limitation of only a few kilometers, bridges are needed to extend the range of communication beyond this limit. Bridges connecting LANs of different types are more complex, in that some minor frame conversion may be required (for example, to bridge an Ethernet to a token ring IAN).

With the emergence of ISDNs, a different kind of bridge was required, to translate between the data frames produced by a LAN and the interface recognized by the ISDN. The traditional data communications bridges were inadequate for this task, because they cannot detect traffic bound for the ISDN and dial an ISDN phone number, and they cannot split up the data frames into segments to support ISDN time division multiplexing. ISDN LAN bridges can take the data frames from a designated host connected to a LAN, and convert these data frames to bit streams suitable for transmission across the ISDN. The protocol has to be converted correctly, and 
control and address information has to be preserved. An ISDN LAN bridge allows multiple connections to a single ISDN line, reducing the need for multiple access devices.

LAN speeds are typically much greater than data rates supported by the ISDN, so the bridge must accommodate this difference. One technique is called inverse multiplexing (bonding), in which a higher speed channel is broken down into parallel lower-speed channels for transmission (the reverse of multiplexing). This technique may also be used to dynamically meet varying bandwidth (capacity) needs.

Terminal adapters (TAs) generally link single non-ISDN terminals to the ISDN. However, a LAN server (front end to all LAN devices) may be tied via a terminal adapter to the ISDN, and an ISDN LAN bridge could serve as a TA.

ISDN LAN bridges may also be called LAN adapters, system adapters, access servers, or half-bridges (since they typically connect with a like partner over a long distance). ISDN LAN bridges may connect PCs, workstations, and file servers, in addition to LANs.

Individual users can dial up access to an ISDN LAN bridge to use the ISDN in the same manner that they would make an ordinary phone call. Devices called digital (high-speed) modems have emerged; modems take one kind of signal (for example, analog) and convert it to another kind of signal (for example, digital) for transmission on the network. A digital modem may or may not provide ISDN LAN bridge services as an enhancement.

The services of an ISDN LAN bridge enable the global networked environment to appear to the user as if that user is operating directly on the remote LAN in a local environment. If there is LAN-LAN interconnection, the system appears as a single LAN to the user. Thus benefits of LAN technology can be extended across the country, or the world.

Routers have additional features beyond those of ISDN LAN bridges. Routers route data between end systems on different subnetworks. An ISDN may be the subnetwork type to which the end system is attached, or it may be an intermediate subnetwork that carries data between the subnetworks that directly serve the end systems (e.g., an ISDN subnetwork can connect two remotely located LANs). Routers perform both the routing and the frame conversion required to connect any two adjacent subnetworks, using non-proprietary protocols such as the Internet Protocol (IP) and the connectionless Network Protocol (CLNP). IP or CLNP routers are built to perform the larger task of connecting any two subnetwork types, and may not be adapted to perform additional services (discussed in sec. 5.2) provided by ISDN LAN bridges. 


\subsection{ISDN LAN Bridge Procurement Considerations}

Given the information above, from a performance or cost standpoint, if users already have or are considering procurement of ISDN access equipment, ISDN LAN bridges may provide an inexpensive alternative which provides multimedia data transfer, relatively high data rates (in increments of $64 \mathrm{Kbps}$ ), and reliable data transfer (generally low error rates on ISDN lines). Furthermore, users can take advantage of evolving technology and position their networks for future growth.

There are alternatives to ISDN use for providing remote access to or from a LAN. Some of these alternatives are leased lines, X.25, switched 56 services, dial-up analog modems, and frame relay. Users should consult appropriate references containing information on these alternatives as a basis for comparison. If the user decides in favor of ISDN LAN bridge functionality, then the remainder of this paper gives more detailed procurement information.

For procuring ISDN LAN bridge functionality, three alternatives are possible. The first alternative is simply to procure an ISDN LAN bridge. A scenario depicting this is given in figure 1 . In this scenario the ISDN LAN bridge is only able to dial a single number to a single destination on the ISDN. Advantages of this alternative are that is it a simpler solution which is less expensive, and provides the services (see sec. 5.2) offered by ISDN access. A second alternative is to procure a "minimal" router, which would include ISDN LAN bridge functionality plus routing extensions. In this scenario, the "minimal" router has to choose between multiple systems connected to the ISDN, and based upon the destination address information it encounters, dial the correct ISDN phone number. Figure 2 illustrates multiple possibilities for paths through the ISDN, but there could also be a single path supporting different destination systems on the ISDN. The advantage of this approach is that it provides more flexibility than the approach above and still provides ISDN services (see sec. 5.2), but it also may be more expensive. A third alternative is to procure a full CLNP or IP router with an ISDN interface. In this scenario, shown in figure 3 , the router can route among a number of subnetwork types (including $\mathrm{X} .25$ ) in addition to providing access to an ISDN. This alternative is the most flexible and provides the most capability, but it is also the most expensive and may contain more functionality than the user needs. This alternative allows direct access to the Internet, but it may not allow all of the ISDN services (see sec. 5.2), because it is general-purpose. Users should choose among these alternatives based on their requirements. If there is no need to distinguish and reach (find) systems across the ISDN, then a routing capability is not needed. However, since in most instances there are multiple systems connected via the ISDN, it is frequently desirable to have ISDN LAN bridge products contain a routing extension. In the remainder of this paper the 


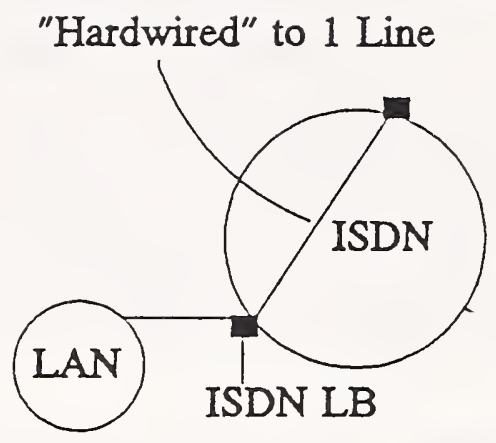

Figure 1

ISDN LAN Bridge

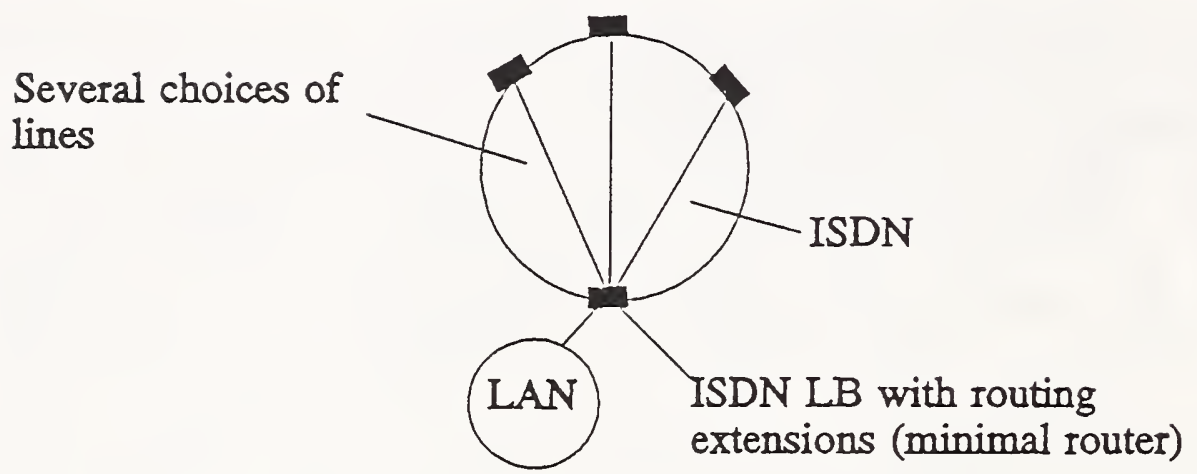

Figure 2

Minimal Router

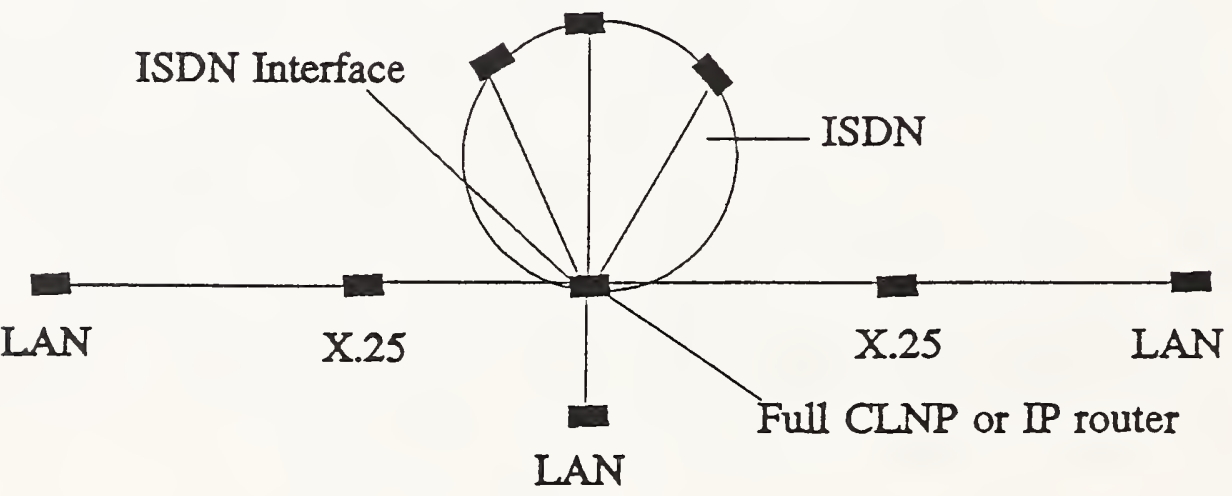

Figure 3

CLNP or IP Router 
term "bridge" will be used to apply to ISDN LAN bridge functionality possible under any of the procurement alternatives mentioned above.

\subsection{ISDN LAN Bridge History and status}

\section{I History of ISDN IAN Bridges}

The need for ISDN LAN bridges grew out of the popularity of LANs as a widespread technology and the recent development of stable ISDN standards and corresponding products. Examination of the current standards for bridges (such as IEEE 802.1) showed that these standards were inadequate or inappropriate for ISDN LAN bridging. Given the user need and the stability of existing standards for ISDN and LANS, preparatory work for ISDN LAN bridging was begun in standards committees such as IEEE 802.9 and in forums such as the North American ISDN Users Forum (NIUF). See section 6 for an expanded discussion on the NIUF.

\subsection{Current status}

Since there were few standards for ISDN LAN bridges, vendors were free to develop their own proprietary products to satisfy their customers' needs. There are ISDN LAN bridge products available today; however, briuges at both ends of the ISDN usually need to be from the same manufacturer. The current product offerings come in several forms, as will be explained below.

There are several groups of vendors which have demonstrated interoperability among themselves. Thus, there are several closed groups or islands of ir eroperability. The position of vendors is that they need to see $x$ business case before developing multivendor interoperable ISDN LAN bridge products.

The position of users is that when they need ISDN LAN bridge technology (as described above) to accomplish their mission, they are considering available services and value. In general, they want non-proprietary solutions in support 0 their functional requirements; they do not want to ke "locked in" to a particular approach of a particular vendor.

The Enterprise Network Data Interconnectivity Family (ENDIF) User's Group of the NIUF is working on multi-vendor ISDN LAN bridge interoperability agreements and plans an interoperability demonstration among various products. Agreements reached on ISDN LAN bridge interoperability generally only apply to North American vendors. Agreements under development in Europe and the Pacific Rim will likely be diffe trom those developed in North America.

ISDN LAN bridge functionality may be implemented in a number of 
ways. However, current offerings generally fall into two classes stand-alone boxes and smart cards for PCs, which are discussed in section 4. Each of these classes contains products from several different vendors. PCs with cards may be able to communicate with a stand-alone box across the ISDN if both are using the same protocol and conventions. However, the relationship is not symmetric between the two classes, in that some of the functions of an ISDN LAN bridge are one-way only.

\subsection{TRIP '92 Status}

The Transcontinental ISDN Project in 1992 (TRIP 192) illustrated a number of uses of ISDN LAN bridge technology, and gave an indication of the status of the technology. The TRIP 192 Atlas gives a report of the activities associated with TRIP '92. The purpose of TRIP ' 92 , conceived by the NIUF, was to demonstrate the achievements and potential of ISDN. Results indicate that this objective was at least partially achieved. IAN to IAN interconnection was demonstrated by several end users. Full network-to-network connections were achieved, so that users on any of the interconnected LANs could easily share the services, applications, or even the computers on any other LAN. Remote access to LANs was demonstrated as well. These demonstrations showed that the ISDN increased bandwidth could serve the needs of many existing and emerging businesses and residential applications.

In one instance, a workstation on one Ethernet LAN and a file server in another Ethernet LAN in a remote location were interconnected using the public ISDN network via $a$ BRI and appropriate ISDN LAN bridges. Another configuration used various bridge/router products with either integrated ISDN interfaces or external terminal adapters to show connectivity between PCs/workstations on separate LANs using ISDN. An ISDN PRI and multiple BRIs to aggregate B channels were both used. The number of $B$ channels actually used was adapted to suit the bandwidth requirements.

In other demonstrations, phonesets and remote fax machines were connected to the bridge. It was also shown how users at single work-at-home workstations could use dial-up access to send and receive information as if they were located at the desk in their office location. Multiple levels of network security were also demonstrated. The TRIP 192 demonstration showed that ISDN LAN bridge products from multiple vendors are here today and they work.

\section{0 ISDN LAN Bridge Product Architecture}

\subsection{Stand-alone Boxes}

The stand-alone boxes generally are offered in either Ethernet or Token Ring versions (a few may support both, or other LAN types, transparently). They typically cost about 500-3000 dollars apiece, 
depending on functionality, number of BRIs supported, etc. They generally tap into the LAN at a predetermined point on the LAN, and are also connected to the ISDN. The box may be small in size (about 1 foot by 1 foot by 3 inches). The functions have previously been implemented in hardware, but many vendors are currently moving to software implementations. These boxes can currently interoperate only with similar boxes also connected to the ISDN. This can be considered one class or instance of interoperability. Prices are subject to change.

Users can configure system parameters, port parameters, and packet parameters. The system parameters include the ISDN switch type, LAN filtering option, and, possibly, the system phone number. There are different kinds of ISDN switches, and not all of them interwork. LAN filtering removes certain LAN addresses from consideration by the bridge. The system phone number is the location of ISDN access.

The port parameters include the called number, and, for CallerID, the allowed receive numbers and origination number. A port is an opening (like a door) for a channel (path) to connect to the device; a port has a particular identifier associated with it. CallerID gives the identification of the caller to the called system.

The packet parameters include specific LAN addresses for filtering and call control. Call control refers to the D channel signalling that supervises the data on the B channels. A LAN address refers to a particular unique location with a LAN.

Many boxes have the ability to "learn" destination packet addresses as they pass through the bridge from the LAN, and to save the address information for use at a later time. The information retained is matched with address information in packet headers passing through the bridge from the ISDN. If there is a match, then the bridge knows where to send that packet on the LAN. If not, then the bridge treats the information as new and retains it for future reference, or may refuse to transmit the packet any further, for security reasons (see 5.6). A few boxes have the ability to exercise a similar "learning" function on packets passing to the LAN from the ISDN.

Boxes have the ability to only forward packets whose destination addresses are in an accepted address list, or to forward any packets except those on a list of blocked addresses. The addresses to be accepted or blocked may be entered manually or learned automatically from the environment. Each bridge should have these parameters set in a manner consistent with other bridges connected to the same ISDN.

A user can specify a phone number to call, port number, data rate, various timers (see below), and the direction of traffic to 
monitor. If traffic exceeds the capacity of one port, then a second port may be automatically opened, to increase bandwidth/speed. The user can manually set, accept or block specified LAN addresses, and can specify the maximum time that an address can stay in the IAN address table.

Users can manually reset addresses, determine whether calls are to be initiated automatically or manually, and set the speed (data rate) of the line. Users can set the retry delay (how long to wait after an unsuccessful call attempt before trying again), and set the timeout interval (amount of time before the line is disconnected if there is no LAN activity on the line).

It may also be possible to set a limited number of protocol options. For transmission, when packets are sent across the ISDN lines, they are encapsulated in a standardized format called a HDLC (high-level data link control) frame. Any other device that recognizes HDLC frames can interoperate with this box, if it uses the same protocol options.

When the channels are operating simultaneously, it is possible for packets to arrive out of order. Most higher-level protocols resolve this ordering problem. A parameter could be set so that packets can be arranged in the order received.

A depiction of the functionality of a stand-alone box is given in figure 4.

\subsection{Smart Cards for PCs}

The other type of device is a set of cards that fits into the chassis of a personal computer (PC) that can be used for LAN-LAN interconnection or PC to I.AN interconnection. Generally, these cards can interoperate with similar cards in other PCs across the ISDN. This is another class of interoperability. The price of the cards may run about 150-1000 dollars depending on functionality. Using this card, the PC may be directly connected to the ISDN switch. Prices are subject to change.

Smart cards inside PCs allow users to configure parameters directly from their terminal screens. A systems administrator is able to set network and node configurations. Network configurations may involve adding, changing or deleting both BRI service and specific site information. There may be a broadcast option (having all sites communicate), and ability to initiate a connection at a specified site (s). Node configuration may involve changing a node name, changing a site, adding a resource or switch type, adding, changing or deleting a line, and adding, changing or deleting a switched service.

Most systems have three or four slots for interface cards; different cards support access to different resources (one card for 


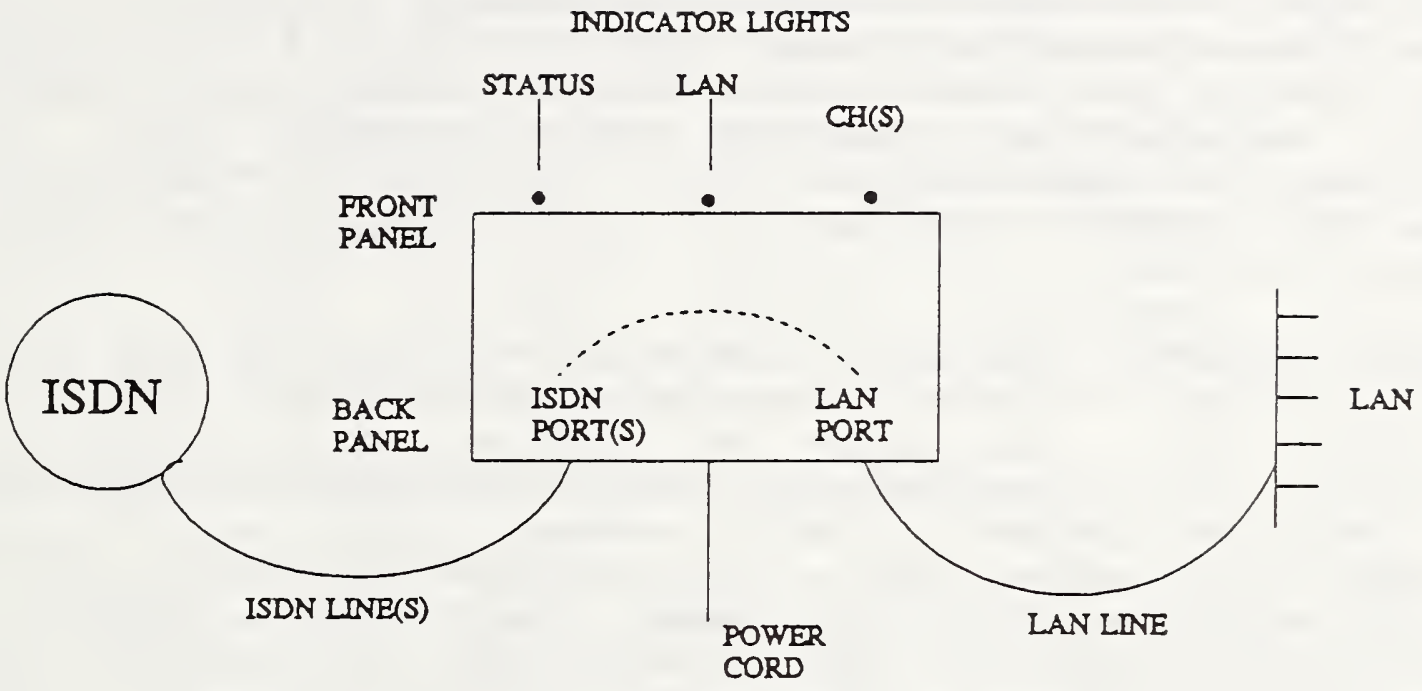

Figure 4

Top-View

Stand-Alone Box

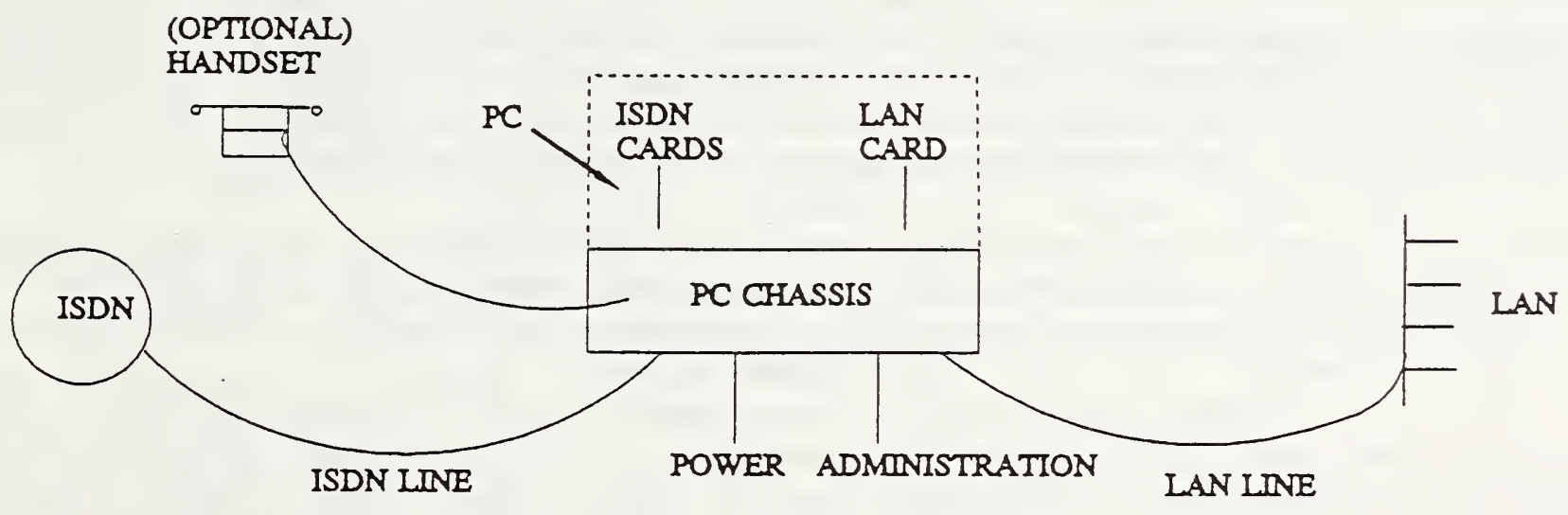

Figure 5

Back View

PC Cards 
LAN access, one for network access, one for individual computer connection, etc.). The user may also be able to specify the type of interface desired (for example, BRI). These products may support a great deal of administrative services and some security services.

Smart cards could combine switched bandwidth (capacity) in 56 Kbps and 64 Kbps increments, could combine switched and leased line access (backup of failed or overloaded leased lines), and may do inverse multiplexing (sending data packets over multiple connections to their destination). In this context "switched" refers to a nondedicated service, and "leased line" refers to a dedicated service.

For their network topology, users may specify carrier services, number of sites, dedicated lines from each site, and switched lines from each site. In terms of application connectivity, the information to be provided may include the number of system nodes, local computing systems (clients, servers, hosts, etc.) attached to each system node at each site, points where local computer systems send and retrieve their data (local client to local server, local client to remote server, host to host, etc.), broadcast paths between sites, and connection paths initiated between sites. For each system in the network, users may identify the following: resource details for each interface card in the system, details on each line attached to the system, and details on each access type (read-only, write, etc.) supported by the system.

Installation may involve powering up the system and administration console, installing system software, and configuring the system (updating a network file, and updating a node file). A network file contains information on network-related components, and a node file contains information on node-related components.

Smart cards provide functions such as a monthly call charge option, a local bridging option, and a bandwidth configuration option. $A$ monthly call charge option allows the user to track call charges on a monthly basis. If the configured monthly limit is exceeded, the system will either inform the user and continue making calls, or inform the user and stop making calls. A local bridging option can support local LAN-LAN bridging at the same time that remote bridging is occurring. A bandwidth configuration option allows the user to customize conditions for automatically adding or dropping connections.

A depiction of the functionality in the smart cards is given in figure 5. In figure 5, the LAN line may not originate from the ISDN card, and an ISDN card may communicate with a generic LAN card on a PC bus. 


\subsection{Summary of Services}

Stand-alone boxes generally represent more sophisticated technology than do cards, can support a greater number of protocols (conventions), and have more flexibility because they present a generic LAN interface. The set of functions provided by PC cards is generally a subset of the set of functions provided by standalone boxes, but in general the services provided by the two classes are moving towards equivalence status. More of the functions in the boxes have been implemented in hardware (but this is changing), so there may be less flexibility in adding new services. In general, for both system types, there is a shortage of acknowledgement information for requests to the systems. In other words, sometimes it is difficult for a user to know whether a specific request completed successfully. Cards may also be integrated with various software packages, such as telecommunications applications software, FAX software, terminal emulators, and desktop conferencing software.

\subsection{User Decisions Regarding ISDN LAN Bridges}

There are a number of important issues about which users must make decisions when procuring and installing ISDN LAN bridges. Users should understand the factors affecting each issue, decide which factors are important to them, and execute their procurement/installation accordingly. Certain factors which may be important to some users may not be important to other users. Some issues are discussed below.

\subsection{ISDN LAN Bridge Interfaces}

The characteristics of the bridge interfaces are important because user applications may have specific transfer requirements and need to know what capabilities to request of the ISDN service provider, including type of connections. These requirements may be dictated by the kinds of information transferred, since ISDN LAN bridge technology can support multimedia applications. The interface considerations which are discussed below are divided into wide area (mostly ISDN) interface concerns, and local interface concerns.

Users should specify the number of B channels desired (i.e., the level of BRI access). If a large volume of traffic is expected, a greater number of $B$ channels may be required. Users should take their throughput requirements (in Kbps) and divide by 64 Kbps (capacity of $B$ channel) to get an indication of their B channel requirements. It is desirable for an ISDN LAN bridge to be "scalable" to a higher level of traffic corresponding to more network connections. A user may dynamically add or drop $\mathrm{B}$ channels accor ing to load. If users anticipate a wide variability in traff : volume over time, the dynamic feature mentioned above might be userul. 
ISDN LAN bridge products may be classified as single port (one port attached) or multi-port (more than one port attached). A port is an "opening" (with a unique identifier) in the bridge through which bits flow; a port can support one BRI, as an example. A multi-port bridge is desirable if larger traffic volumes or different kinds of traffic are desired, but these products are somewhat more complex. A system may have multiple B channels per D channel (subject to BRI and/or PRI constraints), or just two. Note that the $D$ channel is used for signalling, so that as more $B$ channels are used, the requirements on the $\mathrm{D}$ channel to provide signalling capabilities increase. The user may request that the BRI Iines be semantically related (multilink capability). If the ISDN traffic warrants, PRI access may be requested.

Users may need to decide whether rate adaptation is needed for the bridge. Rate adaptation is the upgrading of a lower rate data line (usually 56 Kbps "switched 56" lines) to the 64 Kbps B channel rate of ISDN. There are ITU (formerly CCITT) standards for rate adaptation (for example, V.120). Whether this is needed depends upon the pre-existing data rates of lines coming into the bridge.

An important user concern is the partitioning of BRI and/or PRI lines to handle voice, data, fax, etc. For example, some lines could always be used only for voice, while others could always be used only for data, or the partitioning could be dynamic, depending on traffic characteristics. The ISDN LAN bridge may support multiple ways of accessing BRI and/or PRI service. For example, the user could specify circuit-switched or packet-switched access to the ISDN.

Miscellaneous factors affecting wide-area interfaces are mentioned below. Not all bridges support inverse multiplexing. Users should decide if this feature is important to them. If users have a greater transfer requirement than number of $B$ channels available, inverse multiplexing is one way (on the physical medium) of satisfying this demand in hardware. Another way (using software at a higher functional level) is use of multilink capability. Some bridges support voice-grade (analog) line interfaces in addition to digital (ISDN) interfaces. Different kinds of ports (such as serial ports using RS-232 interfaces) could be configured into the bridge product.

There are other interface issues as well. Cost may influence the kind of ISDN interfaces chosen. For example, if the charges negotiated with the carrier are dependent upon the number of lines opened, it may be more economical to go with a single-port system. Local interface concerns also need to be addressed. A local interface may be attached directly to a IAN host, or to a multiprotocol gateway or router. A multiprotocol gateway is a device that translates one protocol into another protocol if necessary. There are different kinds of LANs, as well as different versions of a particular LAN, and thus, there are different kinds 
of ILAN interfaces to the ISDN IAN bridge. Users need to specify which (and how many) LAN interfaces they want. Another concern is the number of users that can simultaneously access LANs remotely by being connected to the ISDN IAN bridge. Bigger installations may want to support more users simultaneously. A user may wish to connect other devices (or multiple devices of the same type) to the ISDN LAN bridge; examples of such devices include terminals, phones, and fax machines, or PBXs. Such attachments would undoubtedly increase the complexity of the bridge.

\subsection{Supplementary Services}

Supplementary services are standardized ISDN services; they are optional and may be selected based upon user need. Users may request that certain of these be added to the ISDN LAN bridge product. These services are discussed below.

Call forwarding enables the bridge to redirect calls from a specified ISDN number to another ISDN number. There are three types of call forwarding - call forwarding unconditional (the network sends all incoming calls to another number), call forwarding busy (the network sends incoming calls which are blocked to another number), and call forwarding no reply (the network sends incoming calls that meet no reply to another number).

Call hold enables the bridge to interrupt communications on an existing call and then, if desired, reestablish communications. While the bridge has a call on hold, it can set up, connect, or retrieve another call.

Call transfer enables a served user to transform an established call into a call between the other party of the established call and a third party. Call transfer allows a person or machine to transfer a call to a third party during that call; this is different from call forwarding in which an unanswered call is redirected.

Call waiting allows the bridge to be notified of an incoming call $w$ - $i$ an indication that no information channel is available. The s. criber has the choice of accepting, rejecting, or ignoring the wa ring call. This is analogous to the service provided to telephone users.

Completion of calls to a busy subscriber (CCBS) allows a calling party, when encountering a busy destination, to be notified when the busy destination becomes free and causes the service provider to reinitialize the call to the destination party if desired. This may be a particularly valuable service in a bridge product.

Users should be aware that there will probably be extra charges for many of these services. Not all services may be available from every carrier. 


\subsection{Switch Incompatibilities}

There are several common types of ISDN switches in the United states, and not all of them interwork. For signalling purposes, the ISDN LAN bridge needs to know to which kind of ISDN switch it is connected. On some ISDN LAN bridge products there is a user configurable setting or actual physical setting to control the switch identification.

Common switch types include AT \& T and NTI. Bridge users should determine to which switch types their ISDN LAN bridge will be connected and make sure that their bridge products can handle all of the switch types. The more switch types supported, the greater the complexity of the bridge, but also the greater the flexibility of the bridge. Occasionally, switches of the same manufacturer may not interoperate due to incompatible software versions.

\subsection{Address Resolution}

Address assignments should be topology driven if possible. Address formats and user configurations need to be considered in bridge design. When possible, logical in addition to physical addressing should be supported by the bridge, for flexibility reasons.

Bridges need to reconcile ISDN calling and called numbers with specific LAN and local addresses. There may be separate ISDN numbers for voice and data, even if they share a single line. A user may have mobile or portable systems that they wish to attach to an ISDN line. In this case, appropriate address resolution procedures should be defined for such systems. Some bridges actually provide for IP or CLNP address negotiation and resolution. Users should decide if this capability is desired.

The more addressing capabilities are built into the bridge, the more flexible and powerful it will be. However, it will also increase the complexity and cost of the bridge.

\subsection{Compatibility with other Protocols}

Bridges need to support commonly-available protocols for LAN access. Bridges must also support commonly-available modem specifications, as well as commonly-available LAN protocols (such as IEEE 802.3 and 802.5). Dial-up access to the Internet or to an open Systems Interconnection (OSI) network may be desirable in the bridge product. The more protocols supported by the bridge, the more flexible and powerful it is, and the easier it would be for transition and migration involving other network components.

\subsection{Security}

When procuring and installing ISDN LAN bridges, security is important because users want to ensure that data that passes 
through the bridge will have its integrity preserved, be subject to access control restrictions, and be subject to authentication and identification restrictions. For example, it is possible for a bridge to accept calls only from a list of authorized callers, or for a bridge to accept calls only from specific LAN addresses or with specific user passwords. CallerID is a bridge service commonly used to implement the above.

Packet filtering allows incoming or outgoing data packets to be denied access ("filtered out") based on an address in the packet header or a calling number. Packet filtering may be used to put up a firewall to prevent certain networks from unauthorized intrusion.

Password authentication is an important security concern. Users may need authorization to use the ISDN or to access a LAN from the ISDN. The authorization functionality could reside in the ISDN LAN bridge. A list of approved passwords could be kept in the bridge and access denied if the password submitted does not match.

When CallerID is used, the ISDN LAN bridge can record the identification information for network security. The ISDN LAN bridge can also restrict presentation of the identification information to the specific system being called, and not to other systems which would ordinarily have access to the information.

Another security feature is call-back (including call-back on a second line). This service enables the bridge to return a call that a user makes to the bridge for service. This way, the bridge can verify the identity of the user when a line is available.

A document describing ISDN security issues ("Security in ISDN", NIST SP 500-189) is available from the Government Printing office. Users should reference this document if appropriate.

\subsection{Management}

Management in an ISDN LAN bridge product allows users to monitor the performance of their networks (and ISDN LAN bridges). Management involves accessing accounting information, setting configuration parameters, determining performance requirements, and employing traffic (congestion) management.

Accounting information allows a user to monitor the status of a call placed through the ISDN LAN bridge to another device. Did the call complete? A user may also want to know the percentage of time that the ISDN LAN bridge device was utilized, the call status, the time it takes a call to complete, the time it takes a call to set up, and total throughput achieved. The ISDN LAN bridge can be equipped to handle these needs.

Bridges can be configured so that when one B channel is overloaded with data, another B channel could be opened automatically without 
explicit user intervention. A user would probably want user configurable calling capabilities, as well as automatic call setup and teardown based on network traffic and destination address. It is desirable that a bridge product be self-initializing (that is; able to set configuration parameters to default values automatically). It is also desirable to have critical software configured in non-volatile memory (which doesn't get erased when the bridge is disconnected). Bridges can be made to support customer-originated traces of information, as well as automatic recall of critical information.

Users may have special performance requirements for an ISDN LAN bridge, such as the maximum data rate supported, the time it takes to set up a call, the down time percentage of the bridge, and the channel efficiency (avoidance of extraneous bridge loops). Performance requirements may or may not be realistic given the current state of the technology. An important performance (efficiency) enhancement which may be available in some bridge products is link optimization. In link optimization, the bridge is able to distinguish between random LAN "noise" and user data, so that lines are kept open only during actual data transfer. A congestion management feature is the ability of the bridge to handle leased-line backup and overflow for disaster recovery. If such congestion were to exist, critical data would not be lost. Bridges can be made able to sense the level of network traffic, and adjust their line utilization accordingly.

To assist in the management function, some bridges may have Ethernet and other protocol analyzers (which monitor the effectiveness and performance of a protocol or convention) built in. These analyzers can view data in real-time, capture data over time, and save (recall) data from a file. Diagnostic (internal) loopback tests may be helpful in bridge management. Some bridges have the capability to be managed by the simple Network Management Protocol. Remote bridge management is often desirable. Generally, higher speeds involve more complex management. Management features supported by the bridge give the users greater confidence in the performance of the product; however, overhead (non user-data) may be greater and the cost may be higher.

\subsection{Additional Procurement Considerations}

There are a number of additional procurement considerations. For example, users want as many of the services of the ISDN LAN bridge as possible to be transparent. Users would just like to hit a single keystroke on their terminal to set in motion a whole range of activities. Users want their interface to the ISDN LAN bridge to be as simple as possible. Users will want forward compatibility of existing equipment with future purchases. This is a prime concern in this time of fiscal constraint. Setup and installation of ISDN LAN bridge equipment should also be relatively simple. 
Users may procure ISDN LAN bridges with or without data compression. Data compression saves bandwidth and increases the effective data tra :er rate. A disadvantage : the complexity of the algorithms $t h$, perform compression/de npression and the resulting complexi $y$ of the bridge. There are several compression algorithms available offering vãying compression ratios, but currently few have been standardized.

An ISDN IAN brixge must have some knowledge of the network operating systems for LANs interconnected via the ISDN to the bridge. This is beci.se ISDN LAN bricige users may be terminated from access to remote LANs due to network factors intrinsic to those remote LANs, and the ISDN LAN bridge must recognize and react appropriately to provide sufficient connect time and minimize total costs to the user.

Users must consider the type of contract desired for the ISDN LAN bridges that are purchased. The options include direct buy and leasing. Vendors may elect to add optional features at a reduced charge if a arge acquisition is being pursued. There will likely be "fine turing" in negotiations between users and ISDN service providers. Long-distance carriers generall " have stable PRI offerings, but this is not the case with locai phone carriers.

Users should request functionality in ISDN LAN bridge products that will save them money. For example, bridges that work with both analog (voice-grade) and digital networks may save the cost of maintaining duplicate lines. Quick connection establishment and high speeds of transfer tend to reduce costs.

ISDN service providers may charge a flat monthly fee or (more often) may charge a fee based on usage. Typical minimum monthly charges are 50 dollars/month for BRI service, and 500-1000 dollars/month for PRI service, with installation fees of approximately 200 dollars. These prices are subject to change. Users should regotiate for a fee structure that is appropriate for their enviroment and ISDN LAN bridge usage characteristics. It is possible that local carriers could offer a combination of approaches (for example, reduced monthl fee plus a per-minute usage fee, independent of whether voice or data is being carried).

\subsection{ISDN LAN Bridge Interoperability Activities}

Interoperability of ISDN LAN bridge equipment in a multi-vendor environment is an important consideration for users. To address this concern, interoperability activities are taking place under the auspices of the North American ISDN Users' Forum (NIUF). The NIUF was formed in 1988 and is sponsored by the National Institute of standards and Technology (NIST).

The purpose of the NIUF is to create a strong user voice in the injilementation of ISDN and ISDN applications and to ensure that the 
emerging ISDN meets users' application needs. The NIUF should hasten the availability of interoperable, conforming ISDN products and services that meet users' needs.

The reason for the NIUF is that although there are standards for ISDN, the standards are somewhat generic in nature. Therefore, in order for products to be built, additional details need to be specified that allow implementations based on the standards to interwork. The NIUF has created a catalog of national ISDN solutions for selected NIUF applications, and among these is LAN access and LAN-LAN interconnection. Both BRI and PRI uses are supported.

The Enterprise Network Data Interconnectivity Family (ENDIF) is a working group of the NIUF. The ENDIF is currently developing ISDN LAN bridge agreements, and is compiling a record of interoperability testing between pairs of vendors of ISDN LAN bridge equipment. In a recent demonstration, seven vendors participating in this working group have demonstrated fax-like interoperability using an implementation built in accordance with a specification for ISDN LAN bridge interoperability. Both bridging and routing were demonstrated, and Internet access was achieved by the ISDN LAN bridges. The applications supported included downloading files and reading electronic mail. This demonstration showed that in this instance ISDN LAN bridges from different vendors could interoperate when a single ISDN switch type was able to be recognized by all of the bridges involved. This demonstration used a single $B$ channel between devices; future demonstrations will use multiple B channels (multilink capability).

The Internet Engineering Task Force (IETF) is defining protocols for interoperable use of multiple ISDN $B$ channel links between devices. The ENDIF is referencing this work as appropriate, and has used IETF Request for Comment (RFC) documents pertaining to the Point-to-Point Protocol (PPP) (see sec. 2.2) for the abovementioned demonstration. The PPP used the ISDN as the physical layer medium.

Users should closely follow the progress of interoperability described above. Certain interoperability tests may be mandated by a user in the procurement process. However, users should keep in mind that all testing adds to the cost of procurement.

\subsection{Summary and Future Direction}

In sum, the use of ISDN LAN bridges represents an exciting technology which is available to users to provide remote access to LANs and to interconnect LANs across a wide area. This allows users to share resources or operate on a particular LAN or group of LANs as though the LAN (LANs) was local to the user. The economic, functional, and planning benefits will be summarized below. 
ISDN LAN bridge products from different vendors can interoperate, allowing users to pick and choose from a number of different product offerings. Open competition tends to reduce prices. Since charging is usually based on actual usage, use of this technology could save money over other alternatives for wide area networking. The use of bridges for wide area interconnection obviates the need for expensive software on every PC or LAN. Investment in existing fax, modem, analog network, switched 56 service, etc. is protected.

ISDN LAN bridge products offer many functional enhancements not found in other types of offerings. In addition, future offerings are likely to contain even more functional enhancements. The use of a bridge allows one to tap into functionality not available locally, with minimal or no change to the existing user interface.

ISDN LAN bridges can be used in innovative ways to support different applications, such as multiple retrievals from a remote database while voice calls are in progress. The CallerID service of a voice call can be used to locate data in the database. There are other innovative uses of ISDN LAN bridges as well, including support of medical imaging and other healthcare applications, videoconferencing, massive file transfers, photograph transmission, facsimile transmission, telecommuting (work-at-home), production applications (monitoring assembly line from desktop), education/training, and various satellite and residential uses.

The use of ISDN LAN bridges allows a user to plan a structured, organized approach to system development. Various components can be added anywhere in the system without affecting the other components. Users should not lose their investments in hardware and software, and the architecture should be positioned for introduction of new technology at appropriate points.

As ISDN regulatory and availability issues are decided, ISDN hookup will be available to more and more users over time. The popularity of ISDN is increasing in the United States. Some Regional Bell operating Companies (RBOCs) are close to providing ISDN service to all of their subscribers by request. Thus, use of ISDN LAN bridges should become more widespread due to this factor, and service costs should decrease. ISDN may be important in large scale initiatives such as FTS-2000 and other endeavors pertaining to the evolving National Information Infrastructure (NII), so the importance of ISDN LAN bridging will be evident.

Currently the data rates achievable via narrowband ISDN are limited by the physical cable used. In the future, copper cable is likely to be replaced with fiber optic cables, which should greatly increase the available bandwidth. This technology will affect the future design and construction of ISDN LAN bridges, especially since LANs based on fiber optics may support data rates of up to 100 Mbps or higher. Future advances in microelectronic circuitry, as well as the advent of microwave and cellular technology, will 
also affect the design of switching systems and bridges.

Users should make sure that existing bridge products can be removed and replaced in a modularized fashion so that individual components of the network can be examined separately. The transition should be undertaken in a phased, or staged fashion, to minimize the impact on existing services. In the future, it is likely that more sophisticated bridge products will be produced, in support of emerging technologies such as the Asynchronous Transfer Mode (ATM) and Switched Multimegabit Data Service (SMDS) technologies; users should position themselves for the transition to more advanced networking technologies when they become available. 

